\title{
Explaining nutritional habits and behaviors of low socioeconomic status women in Sanandaj: a qualitative content analysis
}

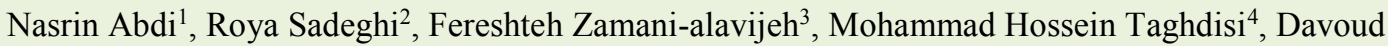 \\ Shojaeezadeh ${ }^{5}$
}

${ }^{1}$ Ph.D. Candidate of Health Education \& Health Promotion, Department of Health Promotion and Education, Faculty of Health, Tehran University of Medical Sciences, Tehran, Iran

${ }^{2}$ Ph.D. of Health Promotion, Associate Professor, Department of Health Promotion and Education, Faculty of Health, Tehran University of Medical Sciences, Tehran, Iran

${ }^{3}$ Associate Professor, Department of Health Education and Promotion, School of Health, Isfahan University of Medical Sciences, Isfahan, Iran

${ }^{4}$ Ph.D. of Health Promotion, Professor, Department of Health Promotion and Education, Faculty of Health, Iran University of Medical Sciences, Tehran, Iran

${ }^{5} \mathrm{Ph} . \mathrm{D}$. of Health Education, Professor, Department of Health Promotion and Education, Faculty of Health, Tehran University of Medical Sciences, Tehran, Iran

\section{Type of article: Original}

\begin{abstract}
Introduction: Health and behavior are closely related subjects because disease is typically rooted in individuals' unhealthy behaviors and habits. This study aims to identify women's nutritional habits and behaviors in order to design interventions to promote nutritional literacy.

Methods: This qualitative research is part of a mixed method (quantitative-qualitative) study, conducted based on content analysis. Data were collected using semistructured interviews, group discussions, and in-depth interviews with married women, aged 18-50 years, who were referred to four health care centers in Sanandaj in 2013-2014.

Results: Nutritional habits and behaviors of participants were classified into two categories: representation of nutritional behavior based on consumption pattern and representation of nutritional behavior based on consumption method. For the former, eight consumption pattern subcategories were formed: meat, dairy, fast food, local foods, fruits and vegetables, soft drinks, and oils. The latter (representation of nutritional behavior based on consumption method), included two subcategories: consumption method in line with health and consumption method inconsistent with health.

Conclusion: Results of this qualitative study provide a solid foundation for development and designing interventions to nutritional literacy promotion based on needs. The designed intervention to healthy nutritional behavior should be based on empowering women and providing facilitator factors of a healthy diet. While designing this study, with a holistic perspective, individual and social aspects of a healthy diet should be taken into account.
\end{abstract}

Keywords: nutritional behavior, nutritional habits, qualitative study

\section{Introduction}

Health and behavior are closely related subjects because most disease is typically rooted in an individual's unhealthy behaviors and habits. Individual behavior is likely to decrease or increase cancer risk, heart attacks, strokes, and similar physical damage (1). Chronic diseases are nowadays considered serious threats to health and longevity in developing countries. The World Health Organization (WHO) reports that $70 \%$ of mortalities in Iran are due to chronic diseases (2). Also in Iran, the highest burden of diseases is related to noncommunicable diseases. Obesity,

\section{Corresponding author:}

Associate Professor Dr. Roya Sadeghi, Department of Health Promotion and Education, Faculty of Health, Tehran University of Medical Sciences, Tehran, Iran. Tel: +98.2166954232, Email: sadeghi_roya@yahoo.co.uk Received: September 14, 2015, Accepted: December 12, 2015, Published: January 2016 iThenticate screening: October 26, 2015, English editing: December 20, 2015, Quality control: January 02, 2016 (C) 2015 The Authors. This is an open access article under the terms of the Creative Commons Attribution-NonCommercialNoDerivs License, which permits use and distribution in any medium, provided the original work is properly cited, the use is non-commercial and no modifications or adaptations are made. 
arterial hypertension, lack of physical activities, hypercholesterolemia, and addiction are five factors of primary risks, which have devoted the largest share to burden of diseases (3). One of the most influential factors in the incidence of disease is unhealthy lifestyle and its transformations. Fifty-three percent of causes of death are associated with individual lifestyles (4). Positive behavior patterns such as proper diet, regular exercise and physical activities, avoidance of alcohol and tobacco consumption play a significant role in individual health promotion (5). Lifestyle modification requires changing behaviors that constitute a major part of daily life habits of individuals (6). Healthy diet and consumer culture are considered as the most prominent factors of lifestyle and the most effective in promoting public health. Generally, good nutrition and healthy eating leads to the overall stable health of society (7). Food habits and culture are interrelated, i.e., nutritional behavior patterns of a society is a part of its cultural patterns. In other words, beliefs, values, and social norms have a role in forming nutritional habits. Day after day, the evidence shows that cultural and social foundations such as nutritional habits, education, and literacy, and awareness and access to information as well as nutritional literacy play a prominent role in shaping healthy nutritional behaviors and decisions. For this reason, over the recent years, more attention has been given to the role of culture and nutritional literacy (8). Consequently, being aware of opinions, demands, needs, preferences, behaviors, and beliefs of customers is regarded as a basic and important principle, which plays a critical role in designing intervention programs to promote healthy eating behaviors. Therefore, relying on conditions and real needs of people and considering their beliefs and sanitary norms, studies that are able to present programs to modify the nutritional patterns of a society are among today's top health priorities (9). Qualitative methods, collecting in-depth data, can assist health trainers to discover the origin of health-related problems and behaviors along with environmental factors from the perspective of those who are directly involved with the problems. These trainers also can achieve a comprehensive approach in this regard (10). Qualitative approaches are capable of familiarizing us with latent layers of behavior. Because nutritional behavior is a series of beliefs and habits that rely on social norms and values, we need to further discover the behavior in its cultural context before creating plans to improve it. Different qualitative studies have been conducted in terms of examining and understanding views (11-13), beliefs and experiences (14), and nutrition teaching (15) as well as barriers to healthy eating behaviors (16, 17). Obviously, forming nutritional beliefs is considered a subculture, which, as with other social phenomena, is of great importance. In conducting exploratory studies regarding healthy diet, therefore, taking sociocultural aspects of forming wrong nutritional beliefs into consideration seems essential. These aspects help health practitioners consider different possibilities in planning and developing training interventions. Focusing on various nutritional behaviors in different cultures and the anonymity of nutritional behaviors and their causes in Sanandaj, first by conducting qualitative research on nutritional behaviors and beliefs, the meaning of healthy food and causes of nutritional behaviors in the target population will be explained. This formative study, conducted for the first time in Iran, will help us to determine appropriate interventions to improve nutrition literacy in women.

\section{Material and Methods}

This research is part of a mixed-method study, which is conducted based on content analysis. The participants were composed of 18- to 50-year-old married women who were referred to four health care centers in Sanandaj, which were selected using purposive sampling. Written informed consent was obtained from all participants who were free to participate in the study or exit the study at any point during the research. The criterion for the adequacy of research sample was data saturation; finally, 50 women covered by a health care center participated in the study. Using semistructured interviews, the data were collected through group discussions and individual in-depth interviews. During five sessions of discussion groups-minimum of six and maximum of 10 people in each group-and eight individual interviews, the data were collected. The time period for discussion groups and individual interviews were between 1 and 2 hours and 1.5 hours, respectively. This time period varied, depending on the condition and process of the sessions or interviews. The interviews were started with raising general questions. For example, how do you define a healthy diet? To maintain a healthy diet, what actions do you take? Afterward and gradually, deeper questions were asked, in line with the research questions. Interviews began in late October 2013 and ended in late June 2014 until data saturation. In the last interview, no new data were obtained. After gathering the data, content analysis was employed. Immediately, after each session, on the day of the interview, all recorded interviews were thoroughly transcribed in detail. To fully understand the situation and get involved in the process of the interviews, the interviews were listened to, and the transcribed data were studied several times by the researcher. Following that, coding (converting the semantic and meaningful units to shorter, understandable, and desired concepts) was completed. To identify similarities and differences, to merge similar codes and to form categories, the codes were reviewed. In the following step, subcategories were classified based on similarities, differences, and appropriateness and categories were extracted as well. Finally, reconsidering categories, subcategories, codes, and data, main themes 
of the study were extracted. MAXQDA Version 10 was employed for coding and classification of the interview texts. To ensure accuracy and reliability of the obtained qualitative data, criteria such as acceptability, reliability, stability, and transmissibility were considered. Long-term involvement of the researcher with the research topic, data, and observing extracted codes and also immediate transcription, detailed record of all steps of the research, and providing a similar position for participants are all considered as approval of acceptability research. Some parts of the interviews, along with extracted codes, categories, and subcategories, were assessed by some observers outside the research team who were familiar with qualitative research methods. Sampling in this study utilized maximum diversity, which helps with transmissibility to increase the results.

\section{Results}

The participants were 50 patients with a mean age of $32.5 \pm 6.0$. Half the women did not have a diploma. Eighty percent of the women worked in the household and others were employed. Nutritional habits and behaviors of participants were classified into two categories: representation of nutritional behavior based on consumption pattern and representation of nutritional behavior based on consumption method. For the former, eight consumption pattern subcategories were formed: meat, dairy, fast food, local foods, fruits and vegetables, soft drinks, and oils. For the latter (representation of nutritional behavior based on consumption method), there were two subcategories: consumption method in line with health (healthy one) and consumption method inconsistent with health (unhealthy one). The majority of participants noted that most of their meals contain meat. They believed that imagining nonmeat meals is nearly impossible, and there are very few non-meat foods they can cook for their meals. One of them said, "All our meals contain meat, even if the amount is little; to us, meat adds flavor to the food. Meat makes food tasty and a free-meat food would be almost tasteless. In most cooking programs on TV, they use meat to train people how to cook different kinds of food. I don't think meat is risky." Most participants reported either low or no fish consumption, which is primarily blamed on the unpleasant smell of fish. The same participant, for example, said, "There is no fish in our diet. My husband and I hate the smell of fish. I have frequently heard on TV that fish is a healthy food and it should be eaten at least once a week, but we just can't because, if I eat fish, I will get sick." The most widely consumed meat mentioned by participants was chicken. They typically believe that its low price and wide variety of foods that include chicken make it a popular meat. A participant confirmed that it is cheaper and lots of food can be cooked using it. He also said, "As I have heard from TV, it is healthier than red meat. If one eats red meat regularly, the risk of heart disease and hypertension will increase. At least chicken is not like that, although most chickens are nowadays sick. All chickens, either lightweight or heavy, contain chemicals. Some believe that the lightweight ones are healthier, but I don't think so! Eating them leads to unwanted hair growth and hormone elevated. However, it is still common and all consume it." Regarding diary consumption patterns, most participants consume them inadequately. Half mentioned that they consume buttermilk and yogurt along with their meals. However, they also mentioned low consumption of milk and whey. Per one participant: "As doctors tell us, three to four glasses of milk should be drunk daily. I think it is impossible. First, who can drink that much; second, it is expensive. Our milk consumption is low. If I drink milk, I will suffer from intestinal pain because my intestine is not compatible with milk. Instead, we consume yogurt a lot. We have it on our table along with most meals. Even my daughter sometimes eats yogurt as a snack but is reluctant to drink milk. She hates it."

In response to fruit and vegetable consumption, most participants noted low consumption, especially for their children; only a small number mentioned consuming fruit besides cake and other refreshments as their snacks. One of the women, complaining about unwillingness of kids in terms of fruit consumption, said, "From time to time, I put vegetables on the table to be consumed with main courses, but my husband eats a little and my kids don't eat. They even don't eat any fruit. I sometimes have a quarrel with them over this. I don't know why lack of eating fruit has been epidemic among children! They don't eat any, neither at home or at parties. However, they eat a lot of junk food such as pastry, cookies, chocolate, chips, cheese puffs, etc."

When asked about fast food, most participants believed that fast food consumption is harmful and causes cancer. However, due to its delicious flavor and quick preparation, they believe its consumption is unavoidable. Therefore, most participants noted consuming fast food once a month. For instance, one said, "I know that the nutritional value of sausage is very low, and it is believed to cause anemia and cancer. Yet, once a month, we eat it: either I make it at home or we go out to it. I can't help eating them, you know, because I really love spicy sausages, especially Hindi ones." Another participant mentioned: "We should include everything in our diet; our body needs everything. Eating fast food one or twice a month will not harm our body that much." Nevertheless, most participants felt that the healthiest dishes are local and confirmed high consumption, especially in fall and winter. One said that, "To me, local foods are the best. I usually cook them, especially in the fall and winter; we often consume them once or twice 
a week. I can say that they guarantee my family's health." Some cooked traditional and local foods such as tarhana, lentils, and frumenty (wheat dish); one participant believed that they clean (purge) inner parts of body. She noted, "Bean soup (consists of wheat, pea, lentil, and red beans), for example, strengthens the body and is almost chemicalfree." Asking about cereal consumption, most participants' replies noted daily consumption of rice. They believed that meals should be accompanied by rice (rice is an Iranian staple food), and there are a few food meals without rice." Another participant noted: "We consume rice almost every day, and I don't think it is unhealthy. We are used to eating it every day. Since we are not diabetics, eating it will not be a problem. We do test yearly and have no serious problem, so no need to reduce the amount of rice."

Another category was the representation of nutritional behaviors based on consumption method, which contained two subcategories: healthy and unhealthy methods of consumption. In terms of healthy methods of consumption, cooked meals instead of fried, more consumption of white meat rather than red, consumption of less fatty dishes, and eating fresh food were mentioned by participants. One participant, in this regard, said, "I think frozen food and leftovers are not healthy. I use enough ingredients while cooking meals so that everything is eaten in that meal. Even when I go shopping, I do not purchase a lot so that I keep them in the refrigerator for a long time." Another participant suggested that, "We used to consume fried foods a lot. Due to the increase number of diseases, however, over the last years I have either cooked the meals or steamed them. At first, my husband complained about it, but after a while he got used to it. He does not like fried food and red meat anymore. Most of the time he buys chicken and fish." On the other hand, in regards to unhealthy consumption, participants mentioned the following: beverage drinking along with high consumption of soft drinks, high consumption of junk food such as crisps and cheese puffs, eating too fast, drinking tea immediately after the meal, and excessive consumption of fried and high-calorie foods. "In our family," according to one participant, "After finishing the meal, we drink tea without delay, although it is believed that it is better to be delayed due to anemia. Drinking tea with a delay gives us a headache." One participant said, "My kids drink water while eating a meal regularly. They, then, drink buttermilk and Coca-Cola, a habit they have learned from their dad. We used to drink Delester with all meals, even fried eggs. My husband and I became too fat, so we decided to reduce the amount of Delester."

\section{Discussion}

The main objective of this study was to identify nutritional behavior through group discussions as well as individual interviews to design and implement interventions to promote nutritional literacy. The collected data through interviews and discussion groups were divided into two main categories: representation of nutritional behavior based on consumption pattern and representation of nutritional behavior based on consumption methods. Women's nutritional behaviors, in this study, revealed dietary patterns and consumption methods. Nowadays, to evaluate the effect of diet on health, nutrition experts use a dietary patterns approach and add such analysis to other traditional analyses (such as dishes, nutrients, and food groups) in this regard (18). Encompassing nutritional behaviors of individuals, analysis (based on dietary patterns) can provide researchers with more information about nutritional etiology of diseases (19). Conceptually, the investigation of dietary patterns is closer to reality because people do not receive nutrients separately, and their varied diet includes different combinations of nutrients, which can interfere with each other or have a synergistic effect (20). On the other hand, investigation and interpretation of foods and specific nutrients seem difficult because there is a strong relationship between different foods and nutrients. In the analysis of dietary patterns, this could be advantageous because dietary patterns are designed based on dietary habits (21). Besides, if nutritional interventions are designed and implemented based on changes in dietary patterns, they will be more complete and easier to implement $(22,23)$. Dietary pattern analysis helps us to make dietary recommendations to patients because, in terms of translating words, they can better understand dietary patterns more than nutritional benefits. Researchers identified two common dietary patterns: healthy and Western. The healthy pattern is rich in vegetables, fruit, beans, whole grains, and fish; Western patterns contain a large amount of meat products, red meat, butter, full-fat dairy products, egg, and cereals (24). Thus far, only one study in the country (Tehran) has been conducted in which common dietary patterns among female teachers were investigated (25). The study employed factor analysis on the data obtained from FFQ and identified three common dietary patterns: healthy dietary pattern contained plenty of fruit, vegetables (especially leafy green vegetables and cabbage), tomatoes, poultry, tea, fruit juice, and whole grains; in the Western dietary pattern, refined grains, red meat, butter, meat products, full-fat dairy products, pizza, potato, hydrogenated vegetable oils, soft drinks, sweets and desserts were abundantly consumed, and vegetables and low-fat dairy products were scarcely consumed. Traditional dietary patterns consisted of a considerable amount of refined grains, potato, whole grains, tea, hydrogenated vegetable oil, cereals, and red meat broth. Findings of the current study shows that dietary behaviors of participants are mainly 
based on Western patterns. High consumption of red meat, refined grains such as rice, high-calorie snacks and sweets, processed meat products as well as low consumption of fruits, vegetables, and whole grains has comprehensively formed the dietary pattern of participants. The similarity between Western dietary patterns and our society is not surprising because, in our country, in terms of lifestyle, physical activity, and diet, fast changes are happening. Urbanization and improving economic conditions are the main reasons for such changes $(26,27)$. Previous studies in terms of Western dietary patterns indicate changes in dietary patterns of people and being away from healthy dietary patterns and their relation with problems such as obesity (28), attention deficit hyperactivity disorder (29), and metabolic syndrome and its components as well as some inflammatory biomarkers $(30,31)$. Research has indicated that observing dietary patterns are a valid method for evaluating the relation of nutrition and diseases, and dietary patterns resulting from statistical methods have a significant relationship with diseases. However, some researchers believe that, in dietary patterns analysis, dietary behaviors such as pattern, time, and number of meals and snacks should be considered (32). Yet, considering the limited information in terms of common dietary patterns in Iran, on the one hand, and the high prevalence of noncommunicable diseases, on the other, dietary patterns might play a significant role in the increasing prevalence of these diseases. Therefore, nutrition researchers are required to first identify these patterns and then investigate their relationship with common diseases in the country and, finally, design interventions to promote healthy eating behaviors based on the dietary pyramid. The Harvard Rebuilt Dietary Pyramid (Harvard Health Book) is a comprehensive guide to design interventions to a healthy diet, which does not have the downsides of the USDA pyramid. At the base of the pyramid, we observe physical activities. It is also advises low consumption of saturated fat, sweets, carbonated beverages, fast and fried foods, potato, and red meat (33).

\section{Conclusions}

Adopting appropriate nutritional strategies such as through the healthy eating pyramid, which is used in this study, can help people's health promotion and the improvement of their nutritional behaviors. Through deep understanding of women's nutritional behaviors and their perspectives on benefits, barriers, and appropriate ways of communication as well as their demands, the results of this qualitative study provides a solid foundation for development and designing interventions to nutritional literacy promotion based on their needs. Because most participants want to learn healthy diet skills, the designed interventions to healthy nutritional behavior should be based on empowering women and providing facilitator factors of a healthy diet. Also while designing, with a holistic perspective, individual and social aspects of healthy diet should be taken into account. Moreover, within the framework of exchange theory, the benefits of healthy behavior should be increased and its barriers should be reduced so that the customer reaches his goal, which is to promote his nutritional behavior and nutritional literacy.

\section{Acknowledgments:}

This paper describes part of the results of research performed for a $\mathrm{PhD}$ degree in health education and health promotion. We hereby thank all people who contributed to this research.

\section{Conflict of Interest:}

There is no conflict of interest to be declared.

Authors' contributions:

All authors contributed to this project and article equally. All authors read and approved the final manuscript.

\section{References}

1) Lee K. Global Health Promotion: How Can We Strengthen Governance and Build Effective Strategies. Health Promot Int. 2006; 21(S1): 42-50. doi: 10.1093/heapro/da1050, PMID: 17307956.

2) World Health Organization. The impact of chronic disease in the Islamic Republic of Iran. 2006; Available from: http://www.who.int/chp/chronic_disease_report/media/impact/iran.pdf

3) Asgari F, Aghajani H, Haghazali M, Heidarian H. Non-Communicable Diseases Risk Factors Surveillance in Iran. Iranian Journal of Public Health. 2009; 38(S1): 119-22.

4) Park J. Health Services Principals. Trans. Shojaee Tehrani H. Tehran: Samat Publication. 2004. P. 42.

5) Delaun SC, Ladner PK. Fundamental of nursing standard and practice. New York: Delmar Thomson Learning. 1998. p. 75.

6) Tashiro J. Exploring health promoting lifestyle behaviors of Japanese college women: perceptions, practices, and issues. Health Care Women Int. 2002; 23(1): 59-70. doi:10.1080/073993302753428438, PMID: 11822559. 
7) Mohseni M.Handbook of Medical Sociology. Tahhoori, 6th Edition, 2001.

8) Navabakhsh M, Mosanna A. An Introductory Evaluation of Nutritional Changes in the Present Societies. Food Technology \& Nutrition. $2012 ; 9(1)$.

9) Ramezani Tehrani F, Farahmand M, Amiri P, Ghanbarian A, Azizi F. Men's Perception Regarding to Healthy Nutrition Inhibitors: a Qualitative Research. 2012; 11: 725-35.

10) Farquhar SA, Parker EA, Schulz AJ, Israel BA. Application of qualitative methods in program planning for health promotion interventions. Health Promot Pract. 2006; 7(2): 234-42. doi: 10.1177/1524839905278915, PMID: 16585146.

11) Allison C. Sylvetsky,Monique Hennink, Dawn Comeau. Youth Understanding of Healthy Eating and Obesity:A Focus Group Study. Journal of Obesity. 2013; 8: 182-8. doi: 10.1155/2013/670295, PMID: 23956844, PMCID: PMC3730149

12) Parker S, Hunter T, Briley C, Miracle S, Hermann J. Formative Assessment Using Social Marketing Principlesto Identify Health and Nutrition Perspectives of Native American Women Living within the Chickasaw NationBoundaries in Oklahoma. J Nutr Educ Behav. 2011; 43(1): 55-62. doi: 10.1016/j.jneb.2010.07.002, PMID: 21216367.

13) Coveney J. Aqualitative study exploring socio-economic differences in parental lay knowledge of food andhealth: implications for public health nutrition. Public Health Nutr. 2005; 8: 290-7. doi: 10.1079/PHN2004682, PMID: 15918926.

14) Dibsdall LA, Lambert N, Frewer LJ. Using interpretative phenomenology to understand the food-related experiences and beliefs of a select group of low-income UK women. J Nutr Educ Behav. 2002; 34: 298309. doi:10.1016/S1499-4046(06)60112-7, PMID: 12556268

15) Gans KM, Lovell HJ, Fortunet R,McMahon C, Carton-Lopez S,Lasater TM. Implications of qualitative research for nutrition education geared to selected Hispanic audiences. J Nutr Educ. 1999; 31: 331-8. doi:10.1016/S0022-3182(99)70486-3.

16) Farooqi A, Nagra D, Edgar T, Khunti K. Attitudes to lifestyle risk factors for coronary heart disease amongst South Asians in Leicester: a focus group study. Fam Pract. 2000; 17: 293-7. doi: 10.1093/fampra/17.4.293, PMID: 10934175.

17) Nakkash R, Soweid RA.A, Nehlawi MT, Shediac-Rizkallah MC, Hajjar TA, Khogali M. The development of a feasible community-specific cardiovascular disease prevention program: Triangulation of methods and sources. Health Educ Behav. 2003; 30: 723-39. doi: 10.1177/1090198103255521, PMID: 14655866.

18) $\mathrm{Hu}$ FB. Dietary pattern analysis: a new direction in nutritional epidemiology. Curr Opin Lipidol. 2002; 13: 3-9. doi: 10.1097/00041433-200202000-00002, PMID: 11790957.

19) Hacobs DR Jr, Steffen LM. Nutrients, foods, and dietary patterns as exposures in research: a framework for food synergy. Am J Clin Nutr. 2003; 78: 508S-513S, PMID: 12936941.

20) Randall E, Marshal JR, Graham S. Dietary patterns and colon cancer in western New York. Nutr Cancer. 1992; 18: 265-76. doi: 10.1080/01635589209514227, PMID: 1296200

21) Wirfalt AKE, Jeffery RW. Using cluster analysis to examine dietary patterns: nutrient intakes, gender and weight status differ across food pattern clusters. J Am Diet Assoc. 1997; 97: 272-9. doi:10.1016/S00028223(97)00071-0, PMID: 9060944.

22) Sacks FM, Obarzanek E, Windhauser MM, Svetkey LP, Vollmer WM, McCullough M, et al. Rational and design of the Dietary Approaches to Stop Hypertension Trial (DASH): a multicenter controlledfeeding study of dietary patterns to lower blood pressure. Ann Epidemiol. 1994; 5: 108-18. doi:10.1016/10472797(94)00055-X, PMID: 7795829.

23) Appel L, Moore TJ, Obarzanek E, Vollmer WM, Svetkey LP, Sacks FM, et al. A clinical trial of the effects of dietary patterns on blood pressure. N Engl J Med. 1997; 336(16): 1117-24. doi: 10.1056/NEJM199704173361601, PMID: 9099655.

24) National Research Council, Committee on diet and health. Diet and health: implications for reducing chronic disease risk. Washington DC: National Academy Press, 1989. doi: 10.1002/ajhb.1310020514, PMID: 25032333.

25) Esmaillzadeh A, Kimiagar M, Mehrabi Y, Azadbakht L, Hu FB, Willett WC. Dietary Patterns and Markers of Systemic Inflammation among Iranian Women. J Nutr. 2007; 137: 992-8, PMID: 17374666.

26) Ghassemi H, Harrison G, Mohammad K. An accelerated nutrition transition in Iran. Public Health Nutr. 2002; 5: 149-55. doi: 10.1079/PHN2001287, PMID: 12027278.

27) Galal O. Nutrition-related health pattern in the Middle East. Asia Pac J Clin Nutr. 2003; 12: 337-43, PMID: 14505998 
28) Aghapour B , Rashidi A, Dorosti-Motlagh AR, Mehrabi. The association between major dietary patterns and overweight or obesity among Iranian adolescent girls. Iranian Journal of Nutrition Sciences \& Food Technology. 2013; 7: 50.

29) Leila Azadbakht, Mohammad H. Rouhani,Ahmad Esmaillzadeh. Dietary Patterns and Attention Deficit Hyperactivity Disorder among IranianChildren. Zahedan J Res Med Sci 2012; 14(2): 55.

30) Esmaillzadeh A, Kimiagar M, Mehrabi Y, Azadbakht L, Hu FB, Willett WC. Dietary patterns, insulin resistance, and prevalence of the metabolic syndrome in women. Am J Clin Nutr. 2007; 85: 910-8, PMID: 17344515 .

31) Sonnenberg L, Pencina M, Kimokoti R, Quatromoni P, Nam BH, D'Agostino R, et al. Dietary patterns and the metabolic syndrome in obese and non-obese Framingham women. Obes Res. 2005; 13: 153-62. doi: 10.1038/oby.2005.20, PMID: 15761175.

32) Tseng M. Validation of dietary patterns assessed with a food frequency questionnaire. Am J Clin Nutr. 1999; 70(3): 422, PMID: 10479207.

33) Willet W. Health with healthy food choices. first ed. jooya publication, 2007; 19-36. 